\title{
Local Existence of Dynamical and Trapping Horizons
}

\author{
Lars Andersson* \\ Albert Einstein Institute, Am Mühlenberg 1, D-14476 Potsdam, Germany, \\ and Department of Mathematics, University of Miami, Coral Gables, Florida 33124, USA
}

Marc Mars ${ }^{\dagger}$ and Walter Simon

Facultad de Ciencias, Universidad de Salamanca, Plaza de la Merced s/n, E-37008 Salamanca, Spain

(Received 9 June 2005; published 9 September 2005)

\begin{abstract}
Given a spacelike foliation of a spacetime and a marginally outer trapped surface $S$ on some initial leaf, we prove that under a suitable stability condition $S$ is contained in a "horizon," i.e., a smooth 3-surface foliated by marginally outer trapped slices which lie in the leaves of the given foliation. We also show that under rather weak energy conditions this horizon must be either achronal or spacelike everywhere. Furthermore, we discuss the relation between "bounding" and "stability" properties of marginally outer trapped surfaces.
\end{abstract}

DOI: 10.1103/PhysRevLett.95.111102

PACS numbers: 04.70.Bw, 04.20.- $\mathrm{q}$

The application of numerical relativity to black hole spacetimes is, together with the role played by black hole thermodynamics as a testing ground for quantum gravity, among the factors that have caused a shift of interest from global properties of black holes such as the event horizon, knowledge of which requires information about the infinite future, towards quasilocal properties. By quasilocal properties one means such properties that can at least in principle be measured by an observer with a finite life span and hence also can be studied during the course of a numerical evolution of a black hole spacetime.

A closed spacelike surface $S$ in a spacetime $\left(M, g_{\alpha \beta}\right)$ is called trapped if future directed null rays emanating from $S$ are converging. If $M$ contains a trapped surface and satisfies the null energy condition and a suitable causality condition, then $M$ is singular [1]. Suppose $M$ is foliated by a family of spacelike Cauchy surfaces $\left\{\Sigma_{t}\right\}$. The apparent horizon, defined as the family of boundaries of the regions containing trapped surfaces in the $\left\{\Sigma_{t}\right\}$, is a quasilocally defined object that plays an important role in black hole thermodynamics as well as in numerical evolutions of black holes. It should be noted, however, that the apparent horizon depends on the choice of the reference foliation $\left\{\Sigma_{t}\right\}$. If sufficiently smooth, the apparent horizon is foliated by marginally outer trapped surfaces (MOTSs) [2]. The latter are defined to have vanishing outgoing null expansion (while the ingoing one is not restricted).

In numerical evolution of black hole spacetimes, it is now standard to avoid the singular behavior of both gravitational field and gauge conditions in the interior of black holes, by excising a suitable region inside the boundary of the black holes, as defined by a collection of MOTSs, from the computational domain. However, tracking a family of MOTSs during an evolution one encounters the occasional "sudden" appearance of new MOTSs and "jumps" of the MOTSs (see, e.g., [3]). It thus becomes important to study such an evolution analytically as far as possible. In this Letter, we prove in Theorem 1 existence of a horizon, i.e., a hypersurface foliated by MOTSs, provided the initial surface $S$ satisfies a natural stability condition, and in Theorem 2 we give causal properties of $H$. The condition of "strictly stably outermost," which is crucial for these results, means that there is an outward deformation of $S$ such that the corresponding variation of the outgoing null expansion is non-negative and positive somewhere (see Definition 2 for details).

Theorem 1: Let $\left(M, g_{\alpha \beta}\right)$ be a smooth spacetime foliated by smooth spacelike hypersurfaces $\Sigma_{t}$. Assume that some leaf $\Sigma=\Sigma_{0}$ contains a smooth marginally outer trapped surface $S$ which is strictly stably outermost. Then, $S$ is part of a smooth horizon $H$, whose marginally outer trapped leaves lie in $\Sigma_{t}$ and which exists at least as long as these marginally trapped leaves remain strictly stably outermost.

In Theorem 2 below we use the same notation as in Theorem 1 , and we denote by $l^{\alpha}$ the null vector for which the expansion $\theta_{l}$ vanishes on $S$. Recall that the null energy condition holds if $G_{\alpha \beta} j^{\alpha} j^{\beta} \geq 0$ for any null vector $j^{\alpha}$, where $G_{\alpha \beta}$ is the Einstein tensor.

Theorem 2: If, in addition to the hypotheses of Theorem 1, the null energy condition holds, the horizon $H$ is locally achronal. If, moreover, $G_{\alpha \beta} l^{\alpha} l^{\beta}>0$ somewhere on $S$ or if $S$ has nonvanishing shear with respect to $l^{\alpha}$ somewhere, then $H$ is spacelike everywhere near $\Sigma$.

The term horizon in this Letter is closely related to dynamical horizons introduced by Ashtekar and Krishnan [4] and to outer trapping horizons introduced by Hayward [5]. The latter two differ from ours first because they impose additional restrictions on the expansion $\theta_{k}$ along the other future null direction $k$. Moreover, while dynamical horizons are spacelike by definition, outer trapping horizons may have any causal character a priori, but they are required to satisfy an additional stability condition, namely, that the variation of $\theta_{l}$ along $k$ is negative. Our condition of strictly stably outermost can be generalized to variations in the outward past null cone $C_{-}$of $S$. 
Hayward's stability condition is then closely related to our stability condition along null directions. Most of the results stated in this Letter, in particular, the existence theorem, extend to the null case. Details will be given elsewhere [6].

Since the location in spacetime of apparent, dynamical, and trapping horizons depends in general on the foliation $\Sigma_{t}$, it is clear that the same applies to the horizons obtained above. However, for generic dynamical horizons, this dependence is limited by the uniqueness results of Ashtekar and Galloway [7]. We also note that the result in Theorem 2 on the causal character of $H$ is stronger than the one known [5] for trapping horizons.

The example illustrated in Fig. 1 will shed light on the possible behavior of our horizons. It shows a horizon (thick line) in a spacetime foliated by spacelike hypersurfaces $\Sigma_{t}$ (thin lines). The horizon separates a region in which the $\Sigma_{t}$ contain outer trapped surfaces (which we call the "trapped region," shaded in Fig. 1) from a region where the $\Sigma_{t}$ are free of them; the intersection "points" with the foliation are MOTSs. Note that Fig. 1 incorporates naturally the observed sudden appearance of MOTSs during the evolution (e.g., point $e$ ). If the numerical analysis looks for globally outermost MOTSs only (as it is usually done), it is clear that they jump (e.g., from $b$ to $e$ ), while they are in fact connected by a horizon interpolating between both which "runs downward" in some places [in the interval $(d$, e)]. Thus Fig. 1 makes compatible a smooth horizon with the jumps observed numerically. Examples such as Fig. 1 can, in particular, be constructed in spherically symmetric spacetimes by choosing the spacelike foliation $\Sigma_{t}$ suitably. Thus one may expect that the situation described by Fig. 1 is typical, though the causal character of the "downward" part of the horizon is yet unclear; see the discussion in [7].

We now introduce some notation needed for the precise statements of our results. All fields and manifolds will be assumed to be $C^{\infty}$ unless otherwise stated. Let $\left(M, g_{\alpha \beta}\right)$ be a spacetime with signature -+++ . Given a spacelike surface $S$ in $M$ we may choose two future directed null fields $l^{\alpha}, k^{\alpha}$. Recall that the variation $\delta_{p} \nu$ of the geometric object $\nu$ defined on a surface $T$ in the direction of the vector $p^{\alpha}$ is defined by $\delta_{p} \nu=\partial \nu / \partial \tau$ for any one-parameter family of surfaces $T_{\tau}$ with $T_{0}=T$ and $p^{\alpha} \partial_{x^{\alpha}}=$ $\partial /\left.\partial \tau\right|_{\tau=0}$. The null expansion $\theta_{l}$ is defined by $\mu \theta_{l}=$ $\delta_{l} \mu$, where $\mu$ is the volume form on $S$. It should be noted that the variation is additive in the sense that, for example, $\delta_{\psi k+l} \theta_{l}=\delta_{\psi k} \theta_{l}+\delta_{l} \theta_{l}$, for some function $\psi$, but in general $\delta_{\psi k} \theta_{l} \neq \psi \delta_{k} \theta_{l}$.

A closed spacelike surface $S$ is called outer trapped (weakly outer trapped, marginally outer trapped) if one of the null expansions, say, $\theta_{l}$, is negative (nonpositive,

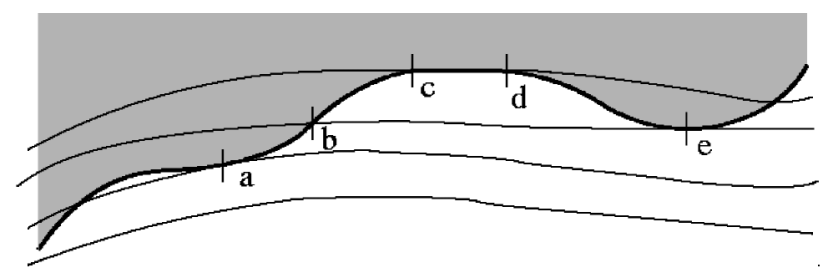

FIG. 1. A horizon.

zero) everywhere on $S$. (For alternative terminology, cf. [8]). The usual definition of "trapped surface" and "marginally trapped surface" requires additional conditions on the expansion with respect to the other null vector $k^{\alpha}$. Corresponding definitions for untrapped surfaces are made by reversing the signs. Let a reference foliation $\left\{\Sigma_{t}\right\}$ of $M$ by spacelike hypersurfaces be given, and select one such surface $\Sigma=\Sigma_{0}$. For a MOTS $S \subset \Sigma$, we define the "outward" direction within $\Sigma$ as the one to which the projection to $\Sigma$ of the null vector $l^{\alpha}$ selected above points. This definition of outward need not coincide with the intuitive one in asymptotically flat spacetimes. However, all our results hold for arbitrary spacetimes (not necessarily asymptotically flat) and $l^{\alpha}$ defines a local concept of outward for MOTSs. The unit outward normal to $S$ tangent to $\Sigma_{t}$ is called $m^{\alpha}$, the future pointing unit normal to $\Sigma_{t}$ is $n^{\alpha}$, and we scale the null vectors $l^{\alpha}$ and $k^{\alpha}$ such that $l^{\alpha}=$ $n^{\alpha}+m^{\alpha}$ and $k^{\alpha}=n^{\alpha}-m^{\alpha}$.

The following definitions are, apart from later use, motivated by similar definitions of Newman [9] and of Kriele and Hayward [2], and by results in these papers.

Definition 1: A marginally outer trapped surface $S$ is called "locally outermost" in $\Sigma$ iff there exists a two-sided neighborhood $U$ of $S$ such that the exterior part of $U$ does not contain any weakly outer trapped surface.

Definition 2: A marginally outer trapped surface $S$ is called "stably outermost" iff there exists a function $\psi \geq 0, \quad \psi \neq 0$, on $S$ such that $\delta_{\psi m} \theta_{l} \geq 0 . S$ is called "strictly stably outermost" if, moreover, $\delta_{\psi m} \theta_{l} \neq 0$ somewhere on $S$.

In Fig. 1, the points in the interval $[a, d]$ represent stably outermost surfaces, those in $[a, c)$ locally outermost ones, and those in $(a, c)$ strictly stably outermost ones. This example and the result in [2] suggest the implications that strictly stably outermost $\Rightarrow$ locally outermost $\Rightarrow$ stably outermost, and the picture also suggests counterexamples for the opposite directions. We now give the tools required to show these results and the theorems.

For a function $\psi$ on $S$, we define a linear elliptic operator $L_{\Sigma}$ by $L_{\Sigma} \psi=\delta_{\psi m} \theta_{l}$. Explicitly, we obtain

$$
L_{\Sigma} \psi=-\Delta_{S} \psi+2 s^{A} D_{A} \psi+\left(\frac{1}{2} R_{S}-s_{A} s^{A}+D_{A} s^{A}-\frac{1}{2} K_{A B}^{\mu} K^{\nu A B} l_{\mu} l_{\nu}-G_{\alpha \beta} l^{\alpha} n^{\beta}\right) \psi
$$

Here $D_{A}$ is the covariant derivative on $S, \Delta_{S}$ is the corresponding Laplacian, $R_{S}$ is the scalar curvature, $K_{A B}^{\mu}$ is the second fundamental form vector [defined by $K_{A B}^{\mu} v_{\mu}=-\nabla_{A} v_{B}$ for any normal $v_{\alpha}$ to $S$, where $\nabla_{\alpha}$ is the covariant derivative on 
$\left.\left(M, g_{\alpha \beta}\right)\right]$, and $s_{A}$ is the torsion of $l^{\alpha}$ (the 1 -form $s_{A}=$ $-\frac{1}{2} k_{\alpha} \nabla_{A} l^{\alpha}$ on $S$ ).

$L_{\Sigma}$ is analogous to the stability operator for minimal surfaces. In general, $L_{\Sigma}$ is not self-adjoint but the eigenvalues of $L_{\Sigma}$ have their real part bounded from below. The eigenvalue with the smallest real part is called the principal eigenvalue. The following holds for second order elliptic operators of the form of $L_{\Sigma}$.

Lemma 1: The principal eigenvalue $\lambda$ of $L_{\Sigma}$ is real. Moreover, the corresponding principal eigenfunction $\phi$ (which satisfies $L_{\Sigma} \phi=\lambda \phi$ ) is either everywhere positive or everywhere negative.

This Lemma is a consequence of the Krein-Rutman theorem which can be applied to second order elliptic operators along the lines in corollary A3 of Smoller [10]. The discussion in Smoller's corollary can be adapted straightforwardly to the case without boundary.

We now restate Definition 2 in terms of $\lambda$ as follows.

Lemma 2: Let $S \subset \Sigma$ be a MOTS and let $\lambda$ be the principal eigenvalue of the corresponding operator $L_{\Sigma}$. Then $S$ is stably outermost iff $\lambda \geq 0$ and strictly stably outermost iff $\lambda>0$.

Proof. If $\lambda \geq 0$, choose $\psi$ in the definition of (strictly) stably outermost as a positive eigenfunction $\phi$ corresponding to $\lambda$. Then $\delta_{\phi m} \theta_{l}=L_{\Sigma} \phi=\lambda \phi \geq 0$. For the converse, we note that the adjoint $L_{\Sigma}^{*}$ (with respect to the standard $L^{2}$ inner product $\langle$,$\rangle on S$ ) has the same principal eigenvalue as $L_{\Sigma}$, and a positive principal eigenfunction $\phi^{*}$. Thus, for $\psi$ as in the definition of (strictly) stably outermost,

$$
\lambda\left\langle\phi^{*}, \psi\right\rangle=\left\langle L_{\Sigma}^{*} \phi^{*}, \psi\right\rangle=\left\langle\phi^{*}, L_{\Sigma} \psi\right\rangle \geq 0,
$$

with strict inequality in the strictly stable case. Since $\left\langle\phi^{*}, \psi\right\rangle>0$, the Lemma follows.

\section{Proposition 1.}

(i) A strictly stably outermost surface $S$ is locally outermost. Moreover, $S$ has a two-sided neighborhood U such that no weakly outer trapped surfaces contained in $U$ enter the exterior of $S$ and no weakly outer untrapped surfaces contained in $U$ enter the interior of $S$.

(ii) A locally outermost surface $S$ is stably outermost.

Proof. The first statement of (i) is in fact contained in the second one. To show the latter, let $\phi$ be the positive principal eigenfunction of $L_{\Sigma}$. Since $L_{\Sigma} \phi>0$ by assumption, flowing $S$ in $\Sigma$ along any extension of $\phi m^{\alpha}$ produces a family $S_{\sigma}, \sigma \in(-\epsilon, \epsilon)$ for some $\epsilon>0$. By choosing $\epsilon$ small enough, the $S_{\sigma}$ have $\left.\theta_{l}\right|_{S_{\sigma}}>0$ for $\sigma \in(-\epsilon, 0)$ and $\left.\theta_{l}\right|_{S_{\sigma}}<0$ for $\sigma \in(0, \epsilon)$. We can now take $U$ to be the neighborhood of $S$ given by $U=\cup_{\sigma \in(-\epsilon, \epsilon)} S_{\sigma}$.

Now let $B$ be a weakly outer trapped surface contained in $U$ which enters the exterior part of $U$. Then, the function $\sigma$ has a maximum $\sigma_{p}>0$ at some point $p$ in $B$. At $p, B$ is tangent to $S_{\sigma_{p}}$ and we have

$$
\left.\theta_{l}\right|_{B} \leq 0<\left.\theta_{l}\right|_{S_{\sigma_{p}}} .
$$

For a surface represented as a graph with respect to a function $f$, the map $f \rightarrow \theta_{l}$ is a quasilinear elliptic operator and the strong maximum principle [11] applies to show that the inequality (2) holds only if $B$ coincides with $S_{\sigma_{p}}$ and hence $\left.\theta_{l}\right|_{B}=\left.\theta_{l}\right|_{S_{\sigma_{p}}}$, which gives a contradiction. Hence $B$ cannot enter the exterior part of $U$. A similar argument applies in the untrapped case.

To show (ii), assume $S$ is locally outermost but not stably outermost. From Lemma 2, the principal eigenvalue $\lambda$ is then negative. Arguing as above, one constructs a foliation outside $S$ with leaves which are outer trapped near $S$, contradicting the assumption.

Theorem 5.1 of Ashtekar and Galloway [7] implies that the domain exterior to $S$ to which outer trapped surfaces cannot enter is determined by the past domain of dependence of any dynamical horizon through $S$, provided that some genericity conditions hold. Outer trapped surfaces "far outside" of a locally outermost MOTS might exist in general [as Fig. 1 suggests for the surfaces in the interval $(b, c)]$. To exclude this, one could define "globally outermost" surfaces (in particular, in an asymptotically flat context). We can now prove our main theorem.

Proof of Theorem 1. Consider a foliation $\Sigma_{t}$ with a MOTS $S$ on $\Sigma=\Sigma_{0}$. Let $C_{+}$be the null cone generated by null rays starting from $S$ in the direction of $l^{\alpha}$ and let $\tilde{S}_{t}=C_{+} \cap \Sigma_{t}$ for $t$ close to 0 . We now introduce coordinates $\left(t, r, x^{A}\right)$ adapted to $\tilde{S}_{t}$ in a neighborhood of $S$ such that at $S, \partial_{r}$ is the normal $m^{\alpha}$ and $\partial_{t}$ is parallel to $l^{\alpha}$.

On $\Sigma_{t}$ we consider surfaces which are given as graphs $r=f\left(x^{A}\right)$ in this coordinate system and we define a functional $\Theta[t, f]$, whose value is $\theta_{l}$ on the surface and which acts on $f$ as a quasilinear elliptic operator of the form $\Theta[t, f]=a^{A B}(f, \partial f) \partial_{A} \partial_{B} f+b(f, \partial f)$, where the coefficients $a^{A B}$ and $b$ are smooth functions depending on $x$ and on $t, f$, and $\partial_{A} f$. For integer $k \geq 0, \alpha \in(0,1)$, let $C^{k, \alpha}$ be Hölder spaces on $S$. Let $I=(-\epsilon, \epsilon)$ for $\epsilon>0$. One checks that for some $\epsilon>0$ and for any $k \geq 2$, there are neighborhoods $\mathcal{U}_{1}$ and $\mathcal{U}_{2}$ of zero in $C^{k, \alpha}$ and $C^{k-2, \alpha}$, respectively, so that $\Theta[t, f]: I \times \mathcal{U}_{1} \rightarrow \mathcal{U}_{2}$ is a well-defined $C^{\infty}$ map. Let $D_{y} \Theta$ be the derivative with respect to the second argument. Then we have, from the definition of $\Theta$, $D_{y} \Theta[0,0] . \beta=L_{\Sigma} \beta$. As $S$ is assumed to be strictly stably outermost, the principal eigenvalue of $L_{\Sigma}$ is positive by Lemma 2. By the Fredholm alternative $L_{\Sigma}$ is invertible as a map $L_{\Sigma}: C^{k, \alpha} \rightarrow C^{k-2, \alpha}$ for arbitrary $k \geq 2, \alpha \in(0,1)$. Now the implicit function theorem for Banach space maps [12] applies to prove local existence of a smooth horizon $H$ which by construction has the property that the leaves $S_{t}=$ $H \cap \Sigma_{t}$ near $S$ are MOTS. By patching charts together it is also clear that existence holds as long as the $S_{t}$ stay strictly stably outermost.

A theorem of Schoen [13] asserts the existence of a MOTS between barrier surfaces $S_{1}, S_{2}$ with $S_{1}$ trapped and $S_{2}$ untrapped if the dominant energy condition holds. Besides its clear interest, this result also suggests an alter- 
native approach to existence of a horizon: Start from a weakly outer trapped surface $S$ on some initial slice and take the null cone emanating from it. By the Raychaudhuri equation, $\delta_{\omega l} \theta_{l}=-\omega W$ for any function $\omega$ and $W=$ $K_{A B}^{\mu} K^{\nu A B} l_{\mu} l_{\nu}+G_{\mu \nu} l^{\mu} l^{\nu}$. If $W>0$, the null cone cuts each subsequent slice on an outer trapped surface which gives the trapped barrier $S_{1}$, while an untrapped barrier $S_{2}$ always exists near infinity for asymptotically flat data. Schoen's result then yields existence of a MOTS on every subsequent slice. The resulting "horizon" may in general jump (e.g., from $b$ to $e$ in Fig. 1), but it need not be outermost. On the other hand, our Theorem 1 requires a MOTS on the initial surface instead of just a trapped one, but we do not assume any asymptotic properties, and we obtain a smooth horizon.

Results on the causal character of the horizon can be obtained by combining again the Raychaudhuri equation on the null cone as sketched above with a maximum principle or a "barrier argument" inside $\Sigma_{t}$ for $t$ near $\Sigma_{0}$. For the latter, we may use, e.g., part (i) of Proposition 1. However, we can also do "both steps at once" by using the following Lemma.

Lemma 3: For a strictly stably outermost surface $S$, any normal variation $\psi m^{\alpha}$ of $S$ with $\delta_{\psi m} \theta_{l} \geq 0$ satisfies $\psi \geq 0$; i.e., the variation cannot be directed to the interior anywhere on $S$. If, moreover, $\delta_{\psi m} \theta_{l} \neq 0$ somewhere then $\psi>0$; i.e., the variation is directed to the exterior everywhere on $S$.

Proof. Let $\phi$ be a positive principal eigenfunction of $L_{\Sigma}$ and define $\chi$ by $\psi=\chi \phi$. A computation shows that

$$
L_{\Sigma} \psi=\chi L_{\Sigma} \phi-\phi \Delta_{S} \chi+2\left(\phi s^{A}-D^{A} \phi\right) D_{A} \chi .
$$

Since $S$ is strictly stably outermost, $L_{\Sigma} \phi>0$ and the strong maximum principle [11] yields that $\chi \geq 0$ if $L_{\Sigma} \psi \geq$ 0 , with strict inequality if $L_{\Sigma} \psi \neq 0$ somewhere.

We can now prove Theorem 2.

Proof of Theorem 2. By construction, the variation of $\theta_{l}$ vanishes along the vector $q^{\alpha} \partial_{x^{\alpha}}=\partial_{t}+\mu \partial_{r}=\omega l+\mu m$ tangent to $H$, where $\omega>0$ is defined by $\partial_{t}=\omega l$. Therefore,

$$
0=\delta_{q} \theta_{l}=\delta_{\omega l+\mu m} \theta_{l}=-\omega W+L_{\Sigma} \mu .
$$

If the null energy condition holds, $W$ is non-negative and under the condition in the second part of Theorem $2 W$ is even positive somewhere. By Lemma 3 it follows that $\mu \geq 0$ in the first case and $\mu>0$ everywhere in the second one, which proves the assertions.

Assume that a 2 -surface $S$ is strictly stably outermost and that the dominant energy condition is satisfied (i.e., that $-G_{\beta}^{\alpha} u^{\beta}$ is future directed for all future directed timelike vectors $u^{\alpha}$ ). Then $S$ is topologically $S^{2}$ (and hence the horizon through $S$ is $S^{2} \times R$ ). We recall here Hawking's argument [14]. Denoting by $\phi$ the positive principal eigenfunction of $L_{\Sigma}$, the stability condition implies that $0<$
$\int_{S} \phi^{-1} L_{\Sigma} \phi$. Then the result follows from (1) after integrating by parts and using the Gauss-Bonnet theorem.

While we have restricted ourselves to local results in this Letter, it would clearly be desirable to determine the global evolution of the horizon in a given black hole spacetime. It would be much more ambitious to look at the global Cauchy evolution for asymptotically flat initial data with a MOTS. In vacuum, one expects this evolution to approach a Kerr spacetime and our horizon to approach the event horizon. Moreover, the area of the marginally trapped slices should approach a quantity not greater than $16 \pi m^{2}$, where $m$ is the mass of the final Kerr black hole. This version of the Penrose inequality [15] would involve the area of the MOTS instead of a minimal surface, and one could, for axially symmetric data, include angular momentum as well [4].

We wish to thank Greg Galloway, Thomas HoffmannOstenhof, José Senovilla, and Helmuth Urbantke for helpful discussions and correspondence. L. A. is supported in part by the NSF, Contract No. DMS 0104402. MM. is supported in part by the Spanish Ministerio de Educación y Ciencia, Project No. BFME2003-02121. W. S. is supported in part by the Austrian FWF, Project No. P14621N05.

*Electronic address: larsa@math.miami.edu

†Electronic address: marc@usal.es

‡Electronic address: walter@usal.es

[1] R. Penrose, Phys. Rev. Lett. 14, 57 (1965).

[2] M. Kriele and S. A. Hayward, J. Math. Phys. (N.Y.) 38, 1593 (1997).

[3] L. Baiotti et al., Phys. Rev. D 71, 024035 (2005).

[4] A. Ashtekar and B. Krishnan, Phys. Rev. D 68, 104030 (2003).

[5] S. A. Hayward, Phys. Rev. D 49, 6467 (1994).

[6] L. Andersson, M. Mars, and W. Simon (to be published).

[7] A. Ashtekar and G. Galloway, gr-qc/0503109.

[8] M. Mars and J. Senovilla, Classical Quantum Gravity 20, L293 (2003).

[9] R. Newman, Classical Quantum Gravity 4, 277 (1987).

[10] J. Smoller, Shock Waves and Reaction-Diffusion Equations (Springer, New York, 1994), Sec. 11, Appendix C.

[11] D. Gilbarg and N. S. Trudinger, Elliptic Partial Differential Equations of Second Order (Springer, New York, 1983), Theorem 3.5.

[12] S. Lang, Differential Manifolds (Springer, New York, 1985).

[13] R. Schoen (unpublished).

[14] S. W. Hawking, in Black Holes, Proceedings of the Les Houches Summer School, Session 1972, edited by C. DeWitt and B.S. DeWitt (Gordon and Breach, New York, 1973).

[15] H. Bray, J. Diff. Geom. 59, 177 (2001); G. Huisken and T. Ilmanen, J. Diff. Geom. 59, 353 (2001). 